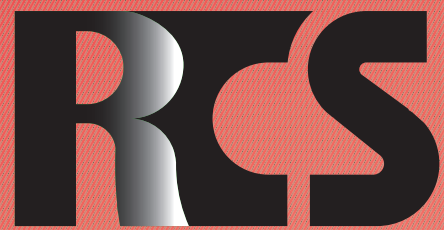

Depósito legal ppi $201502 Z U 4662$

Esta publicación científica en formato digital es continuidad de la revista impresa Depósito Legal: pp $197402 Z$ Z789

- ISSN: 1315-9518 • ISSN-E: 2477-9431

Revista de Ciencias Sociales

Universidad del Zulia. Revista de la Facultad de Ciencias Económicas y Sociales

Vol. XXVII,

No. Especial 3, 2021

Esta publicación científica en formato digital es continuidad de la revista impresa Depósito Legal: pp $197402 Z$ Z789

ISSN: $1315-9518$ 


\title{
Modelos de innovación en la producción de café en la Sierra Norte de Puebla-México*
}

\author{
Barrera Rodríguez, Ariadna** \\ Ramírez García, Adán Guillermo ${ }^{* * *}$ \\ Cuevas Reyes, Venancio ${ }^{* * *}$ \\ Espejel García, Anastacio ${ }^{* * * *}$
}

\section{Resumen}

El café es un cultivo de gran importancia en la Sierra Norte de Puebla, debido al número de productores que dependen de él y por ser la principal fuente de ingresos; forma parte de la cultura productiva mediante el saber hacer que se ha trasmitido de generación en generación. El objetivo fue analizar los modelos de innovación productiva y comercial de los productores de café. Se consideraron las bases de datos del gobierno local; se definió una muestra de 83 productores mediante un muestreo simple aleatorio; se consideraron 46 innovaciones, agrupadas en nueve categorías: Nutrición, control de maleza, sanidad, manejo sostenible de recursos, mejoramiento genético, establecimiento y manejo de la plantación, cosecha, administración y organización. Se realizó una estratificación de productores para definir los modelos de innovación. Los resultados sugieren 4 modelos de innovación; el modelo 4 registró un mayor índice de innovación, en cosecha, control de maleza, establecimiento, manejo de plantación y sanidad. Los modelos 2 y 3 mostraron un bajo índice en nutrición, sanidad, manejo sostenible de recursos, administración y organización. Las variables que inciden en el consumo de café son: Escolaridad, ingreso, precio y etiqueta; otro grupo de consumidores consideran al sabor y aroma atributos de diferenciación.

Palabras clave: Innovación; producción; comercialización; consumidor; café.

Proyecto financiado con recursos de Universidad Autónoma Chapingo mediante el proyecto 20077-C68: Estrategias de valorización y comercialización de productos tradicionales.

** Doctora en Problemas Económico-Agroindustriales. Profesora-Investigadora del Posgrado en Desarrollo Rural Regional. Centro Regionales. Universidad Autónoma Chapingo, México. Pertenece al Sistema Nacional de Investigadores-CONACYT. E-mail: ariadna.barrera@gmail.com (iD ORCID: https://orcid.org/0000-0001-7352-1091

**** Doctor en Geografía. Profesor-Investigador de Centro Regional Universitario Noroeste. Universidad Autónoma Chapingo, México. Pertenece al Sistema Nacional de Investigadores-CONACYT. E-mail: aguillermoramirezgarcia@gmail.com (iD) ORCID: https://orcid.org/0000-0002-1711-5942

***** Doctor en Problemas Económico Agroindustriales. Magister en Economía de los Recursos Naturales y el Medio Ambiente. Economista Agrícola. Investigador del Instituto Nacional de Investigaciones Forestales, Agrícolas y Pecuarias. INIFAP-CEVAMEX, México. Pertenece al Sistema Nacional de Investigadores-CONACYT. E-mail: cuevas.venancio@inifap.gob.mx (iD) ORCID: https://orcid. org/0000-0001-9946-3942

****** Doctor en Problemas Económico-Agroindustriales. Profesor-Investigador del Posgrado en Ciencia y Tecnología Agroalimentaria. Universidad Autónoma Chapingo, México. Pertenece al Sistema Nacional de Investigadores-CONACYT. E-mail: anastacio.espejel@gmail.com (iD) ORCID: https:// orcid.org/0000-0002-6462-6681 


\title{
Innovation models in coffee production in the Sierra Norte de Puebla-México
}

\begin{abstract}
Coffee is a very important crop in the Sierra Norte de Puebla, due to the number of producers who depend on it and because it is the main source of income; It is part of the productive culture through knowhow that has been transmitted from generation to generation. The objective was to analyze the productive and commercial innovation models of coffee producers. Local government databases were considered; A sample of 83 producers was defined by simple random sampling; 46 innovations were considered, grouped into nine categories: Nutrition, weed control, health, sustainable resource management, genetic improvement, establishment and management of the plantation, harvest, administration and organization. A stratification of producers was carried out to define the innovation models. The results suggest 4 models of innovation; Model 4 registered a higher innovation index, in harvest, weed control, establishment, plantation management and health. Models 2 and 3 showed a low index in nutrition, health, sustainable resource management, administration and organization. The variables that affect coffee consumption are: Schooling, income, price and label; another group of consumers consider flavor and aroma attributes of differentiation.
\end{abstract}

Keywords: Innovation; production; marketing; consumer; coffee.

\section{Introducción}

El café es un cultivo de gran importancia productiva, social y económica a nivel mundial; en México, ocupa una superficie de 717.388 hectáreas, lo que representa una producción de 838.997 toneladas, aportando el $2 \%$ de la producción mundial (Servicio de Información Agroalimentaria y Pesquera [SIAP], 2018). Su importancia se estima no solo por el valor de la producción, sino por su aportación ambiental mediante el establecimiento de sistemas agroforestales (manejo con sombra) y captura de carbono, la generación de ingresos en las localidades que corresponden con las de alta marginación.

La producción de café, se realiza mediante policultivo tradicional, se planta en asociación con pimienta, frutales (mango, plátano, zapote negro, naranja), árboles maderables (encino, cedro rojo, caoba), maíz, y frijol, lo cual proporciona servicios ambientales e ingresos económicos para la población (Escamilla, et al., 2005).

Por su valor comercial es uno de los principales productos agrícolas, con una participación importante en el comercio mundial y produce ingresos anuales superiores a los 15 mil millones de dólares para los países exportadores y da empleos directos e indirectos a poco más de 20 millones de personas dedicadas al cultivo, transformación, procesamiento y comercialización del producto en todo el mundo (Canet, et al., 2016, p.1).

Esta cadena productiva es una de las más importantes en el sector agroindustrial del país y destaca por el número de productores que incorpora (504.372 productores) y el número de empleos que genera (Fideicomisos Instituidos con Relación a la Agricultura, 2016), "más de 3 millones de ocupaciones, de las cuales el $70 \%$ las realizan productores y familias de comunidades cafetaleras indígenas, entre las cuales destacan: Zapotecos, mixtecos, mixes, mazatecos, totonacas, nahuas, otomíes, tzotziles, zoques, tojolabales, huicholes y chatinos" (Instituto Nacional de los Pueblos Indigenas [INPI], 2018, p.24).

El café que se produce en México, es mayormente de la especie Coffea arabiga 
L., que constituye el $97 \%$ de la producción nacional, representada por las variedades Typica (criollo, nacional o arábiga), Bourbon, Caturra, Mundo Novo, Garnica, Catuai, Pluma Hidalgo y Maragogype, el 3\% de la producción corresponde a la especie Coffea canephora Pierre ex Frohener, conocida como robusta, cultivada en zonas bajas de Veracruz (principalmente en los municipios de Tezonapa y Tepatlaxco), Chiapas (sobresale el municipio de Cacahoatán) y Oaxaca (regiones de Tuxtepec y Valle Nacional) (Fundación Produce Chiapas y Tecnológico de Monterrey, 2003).

Las regiones cafetaleras en México se ubican altitudinalmente entre los 250 y los $1.500 \mathrm{msnm}$, aunque excepcionalmente se encuentran algunas áreas fuera de estos límites. El mayor porcentaje de cafetales se ubica arriba de los $700 \mathrm{msnm}$, lo que, aunado a factores de baja luminosidad, temperaturas frescas, precipitación suficiente y efectos de latitud, favorecen la fructificación, crecimiento y calidad del café (Santoyo, et al., 1994).

La producción primaria, café cereza y/o pergamino, es el principal eslabón de la cadena productiva para la agroindustria. Posteriormente, el valor agregado permite conservar sus atributos físicos y sensoriales; y finalmente, la comercialización vincula al productor con el consumidor que está dispuesto a pagar por un producto con determinadas características. La materia prima cumple con los estándares de calidad que exige la agroindustria y paralelamente está alineado con la demanda del consumidor final. Por lo tanto, el proveedor (productor), es uno de los actores clave quien garantiza no sólo un buen manejo del cultivo sino un adecuado beneficiado del aromático, ambos procesos determinaran en gran medida la calidad del café.

La innovación, se ha convertido en un concepto de uso cada vez más frecuente, a partir de la aceptación de que un esfuerzo sostenido en la generación y difusión de la misma resulta hoy un factor clave, tanto para mejorar la competitividad de las empresas, como para favorecer un desarrollo en los territorios, en términos de crecimiento económico, y de una perspectiva de calidad de vida de la población (Méndez, 2006; Velázquez, Cruz y Vargas, 2018; Navarro-Caballero, et al., 2020; Vega, Bautista y Martínez, 2020).

Las innovaciones tecnológicas son aquellas actividades o procesos que incorporan nuevas alternativas técnicas en la producción, orientadas por la existencia o identificación de oportunidades de mercado o necesidades de la población (Organización de Cooperación y Desarrollo Económicos [OCDE] y EUROSTAT, 2005; Alburquerque, 2008; Leal, 2012). Algunos estudios establecen que, entre los factores que influyen positivamente en el cambió técnico y uso de innovaciones se encuentra el contacto con casas comerciales que distribuyen productos para el campo (Galindo, 1995), la provisión y uso de crédito y financiamiento, contacto con instituciones del sector agropecuario, factores socioeconómicos de los productores, relación con los extensionistas, exposición a medios de comunicación (Galindo, 2007), y un estudio reciente identificó al mercado como factor determinante en la adopción de innovaciones en cadenas agroalimentarias específicas (Espejel, et al., 2014).

La innovación es un proceso social y territorial, en el cual los usuarios de conocimiento interactúan con los productores de conocimiento e innovación (Alburquerque, 2008). Por lo tanto, el objetivo del presente trabajo es analizar los modelos de innovación productiva y comercial de los productores de café de la Sierra Norte de Puebla-México, con la finalidad de caracterizarlos y definir procesos de transferencia de tecnología.

\section{Metodología}

El tipo de investigación fue de tipo cuantitativa, basada en el diseño de una entrevista semiestructurada (Blasco y Otero, 2008). El estudio se realizó en las regiones Sierra Norte y Nororiental en los municipios de Tepango de Rodríguez, Camacoautla, Ahuacatlán, Ixtepec, Zapotitlán de Méndez y Cuetzalan del Progreso del Estado de Puebla, 
México. En estas regiones se concentra la mayor superficie de producción de café en Puebla, debido a la ubicación geográfica y a las condiciones físico-climáticas y ambientales que favorecen el desarrollo y producción del aromático. De acuerdo con datos del Servicio Nacional de Sanidad, Inocuidad y Calidad Agroalimentaria (SENASICA) y Secretaria de Agricultura, Ganadería, Desarrollo Rural, Pesca y Alimentación (SAGARPA) (2017), en la Sierra Norte se registran 42.591 ha de superficie sembrada, con un total de 22.875 productores; y en la Sierra Nororiental, 23.956 ha y 13.644 productores de café.

Para el análisis del sistema cafetalero, se realizó una encuesta a 83 productores con un universo de 300 , por medio de un muestreo aleatorio simple (Ecuación 1):

$$
n=\frac{N Z^{2} p q}{d^{2}(N-1)+Z^{2} p q}
$$

Dónde: $\mathrm{n}=$ Número de productores que realizaron la encuesta; $\mathrm{N}=$ Número total de productores; $\mathrm{d}=$ Precisión (expresada en porcentaje: $10 \%)=0.1 ; Z=$ Para poblaciones $>100$, la probabilidad de confianza se puede estimar a un $95 \%=1.96$, o bien, a un $90 \%$ $=1.64$. Para poblaciones $<100$, es necesario recurrir a los valores de la distribución $t$ de Student; $\mathrm{q}=$ Diferencial de $\mathrm{p}(1-\mathrm{p})=0.5 ; \mathrm{y}, \mathrm{p}=$ Proporcional de la población $=0.5$.

Se elaboró una encuesta dirigida a los productores, estructurada en cuatro apartados: 1) Información socio-económica, 2) organización de productores, 3) información técnico- productiva, y 4) adopción de innovaciones. Se realizó una visita a la Cooperativa Tosepan Titaniske, ubicada en el municipio de Cuetzalan del Progreso, con el propósito de identificar modelos de organización y comercialización exitosos. El trabajo en campo se realizó de abril-junio de 2018.

\subsection{Análisis cuantitativo}

Se definieron 46 innovaciones agrupadas en nueve categorías: Nutrición, control de maleza, sanidad, manejo sostenible de recursos, mejoramiento genético, establecimiento y manejo de la plantación, cosecha, administración y organización. Las innovaciones se definieron con base en la revisión de literatura (Escamilla, 1993; Inca Rural, et al., 2008; Cuya, 2013; Escamilla, et al., 2015; Santoyo, et al., 2016) y de las visitas a las plantaciones de café. Con base en la información obtenida de las encuestas, se calculó el índice de adopción de innovación (InAI), el cual expresa las innovaciones que realiza cada productor dentro del total de las innovaciones (Muñoz, et al., 2007), y se muestra en la Ecuación 2:

$\operatorname{In} A I=\left(\frac{\text { Innovaciones realizadas por el innovador } i}{\text { Máximo número de innovaciones por los productores } j}\right) * 100$

Se realizó un análisis de clúster o conglomerados y un análisis de componentes principales con el software XLSTAT, con base en las variables de adopción de innovación, con el objetivo de identificar estratos de productores y caracterizarlos en función de las prácticas que realizan en la producción de café. Con la finalidad de conocer las fuentes de innovación del productor, se efectuó un estudio de redes sociales empleando el software UNICET y Key Player 2, se calcularon los indicadores de densidad y centralidad (Rendón, et al., 2007; Aguilar-Gallegos, et al., 2016). Para ello, se realizó una pregunta directamente al productor: ¿De quién aprendió a producir el cultivo del café o alguna innovación agrícola? Con la información proveniente de los productores de café, se llevó a cabo el análisis para definir la red comercial del café cereza y pergamino, empleando la pregunta ¿a quién vende su cosecha?

De igual manera, se realizó y aplicó una encuesta a 120 consumidores en la región de estudio y en la Expo Café 2018, del 30 agosto al 1 de septiembre, realizado en el World Trade Center (WTC), en la Ciudad de México. 
La encuesta tuvo como propósito analizar factores que inciden en el consumo de café mexicano y sus preferencias y con ello definir una estrategia de comercialización.

\section{Resultados y discusión}

\subsection{Adopción de innovación en la producción de café}

El análisis indica que la adopción de innovación es baja, registrando un valor promedio de 35\%. Los índices de menor valor fueron: Administración (Adm), sanidad (San), organización (Org), reproducción y mejoramiento genético (RyMG), nutrición
(Nut) y manejo sostenible de recursos (MSR), quienes registraron valores menores al $30 \%$ (ver Gráfico I). Las categorías de innovación de mayor adopción por los productores analizados fueron, el control de maleza (CM) $(65 \%)$, cosecha (Cos) $(56 \%)$, los productores tienen conocimientos sobre los criterios para la recolección del café. El InAI de establecimiento y manejo de la plantación (EyMP) registró $42 \%$, los productores consideran importante realizar el manejo de sombra, poda de los cafetales y renovación de los cafetales, este último se viene practicando a partir de la incidencia de la roya en el año 2012, con efectos negativos en la producción (Escamilla, 2016).

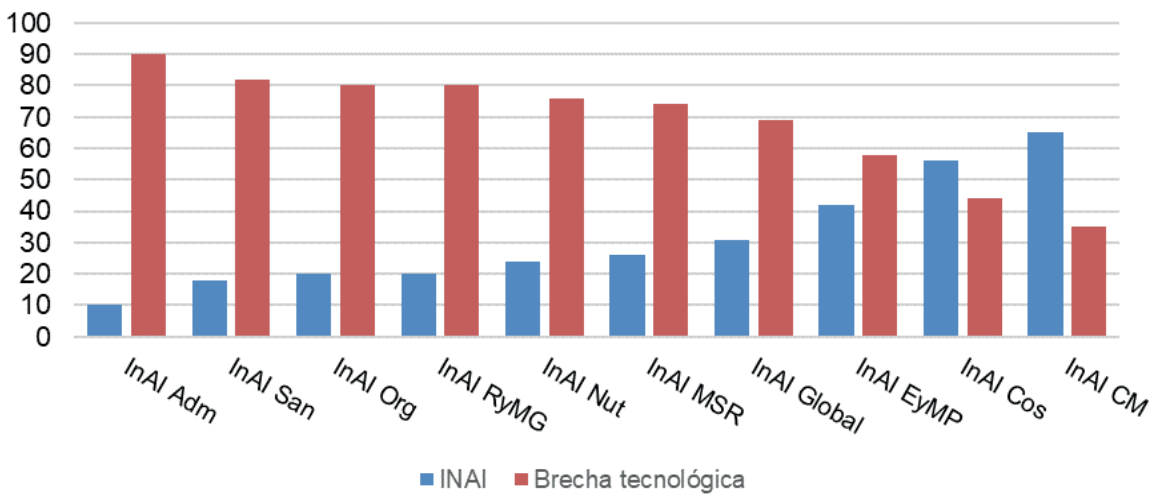

Fuente: Elaboración propia, 2020 con datos de campo, 2017.

\section{Gráfico I: Índice de adopción de innovación por categoría}

El InAI de Nutrición, tuvo un nivel de adopción de $24 \%$, la mayor parte de los productores realiza fertilización en sus cafetales, pero no realiza un análisis del suelo y foliar para la determinación del tipo de fertilizante y dosis de aplicación. El principal fertilizante químico que aplican los productores es el sulfato, por su bajo costo. El $42 \%$ de los productores realiza una sola aplicación de fertilizante, el 30\% realiza dos aplicaciones. La época de aplicación varía entre cada productor, puesto que esta se realiza de acorde a los ingresos individuales, no como un beneficio para la planta y mejora de la producción. Para el InAI de manejo sostenible de recursos, la adopción fue de $26 \%$.

Asimismo, el InAI con menor porcentaje fue el de administración (10\%), 
los productores no llevan un registro sobre los costos e ingresos que obtienen a partir del café, además, no cuentan con un calendario para fijar las prácticas de labores culturales. El InAI de Sanidad, también obtuvo un valor bajo con $18 \%$, se considera que solo los productores que cuentan con el apoyo de SAGARPA (actualmente SADER-SENASICA) no pertenecen a una organización, realizan el control de roya que consiste en aplicar productos fungicidas o el manejo del tejido productivo (poda) en las áreas afectadas.

El InAI de organización, reproducción y mejoramiento genético, obtuvieron un valor de $20 \%$, en ello destaca que un bajo porcentaje de los productores entrevistados pertenece a una organización quien les brinda capacitaciones y talleres para el manejo adecuado del café, además de apoyar en la compra y comercialización del aromático a un precio justo; la mayoría de los productores $(82 \%)$ no pertenecen a ninguna organización debido a la falta de tiempo, o no saben cómo integrarse y trabajan de manera individual, por lo que carecen de apoyo o algún beneficio incluso de las instituciones gubernamentales.

Con respecto a la reproducción y mejoramiento genético, se detectó que los productores organizados cuentan con viveros de café y que ellos mismos realizan la selección de la semilla, solo 2 productores mencionaron que esta innovación la aprendieron del Instituto Mexicano del Café (INMECAFE) en 1974. El 48\% de los productores entrevistados adquieren plántulas de café basándose en la variedad, sin considerar otras características.

Existe una brecha tecnológica elevada entre la adopción de innovaciones, de acuerdo con Muñoz y Santoyo (2010), esta indica la existencia de grupos de agricultores que ya están haciendo las cosas de manera diferente y mejor que sus pares. Es decir, los innovadores no se encuentran en el exterior de las redes, sino que ya son miembros de una comunidad productiva concreta. Con el análisis de clúster se identificaron cuatro estratos de productores con base en el índice de adopción de innovación (ver Figura I).

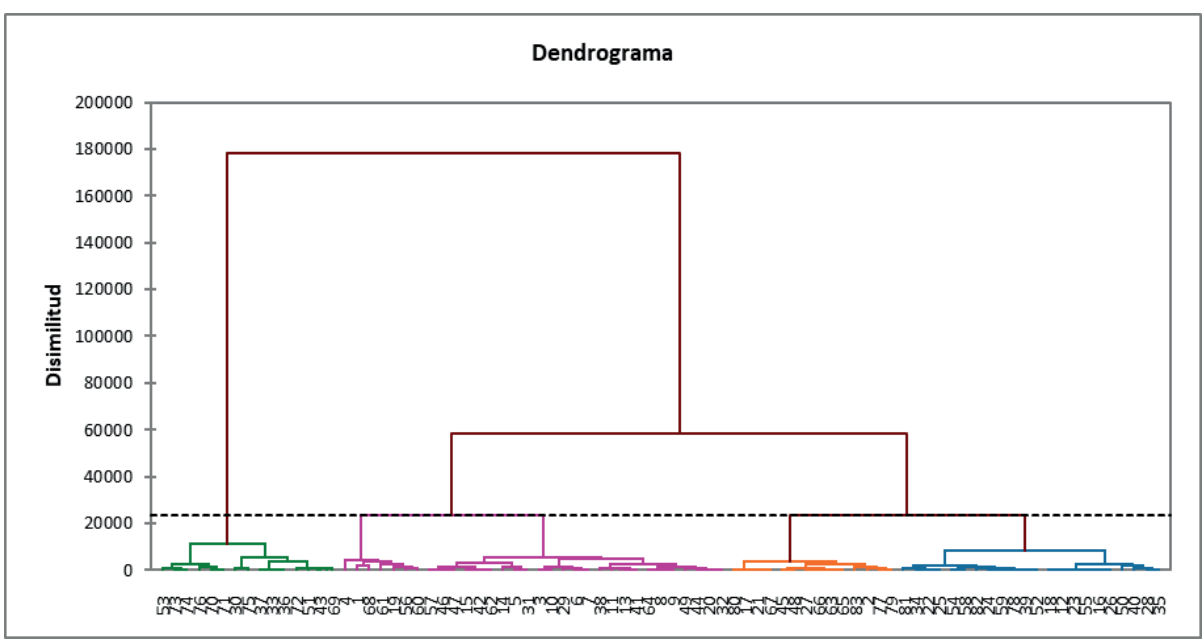

Fuente: Elaboración propia, 2020 con datos de campo, 2018.

Figura I: Análisis de conglomerados (clúster) de productores 
En el estrato 4 se ubicaron los productores organizados, quienes pertenecen a la Cooperativa Tosepan Titaniske y registraron un mayor InAI considerando su constante capacitación en distintas áreas. Mientras que los estratos 1, 2 y 3 (de izquierda a derecha), los productores trabajan de manera individual. El estrato 1, tiene un alto índice de adopción, pero menor al estrato 4. Para los grupos 2 y 3 , la adopción de innovación tecnológica es muy baja.

La organización es un componente de estímulo que reciben los individuos para concebir nuevas ideas y de la adopción o rechazo de la misma va depender la capacidad de la empresa para adaptarse (Muñoz y
Santoyo, 1996). Los productores organizados acceden a procesos de capacitación con mayor frecuencia en diversos temas para el buen manejo agronómico del cultivo, el beneficiado del café y de los estándares de calidad que debe cumplir el aromático (Santoyo, et al., 1994, Aranda, González y Reyes, 2017).

En contraste los productores de los estratos 1, 2 y 3 trabajan de manera individual, en el Gráfico II, se observa que el estrato 1 es el que tiene mayor índice de innovación en diferentes categorías. El menor nivel de adopción, está asociado a factores como el nivel de organización y el acceso a apoyos gubernamentales.

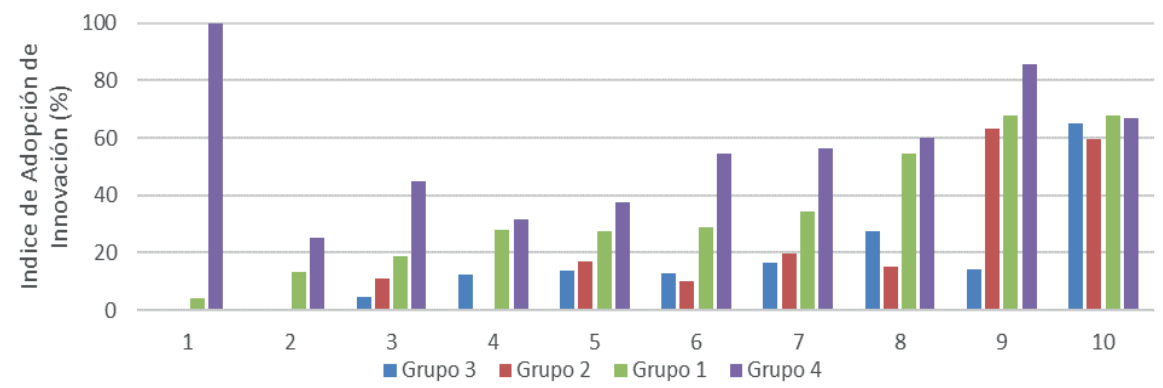

Fuente: Elaboración propia, 2020 con datos de la encuesta 2017.

\section{Gráfico II: Adopción de innovación por categoría de los cuatro estratos}

Las innovaciones de control de maleza, reproducción y mejoramiento genético, fueron las primeras innovaciones que el productor adoptó en 1964, transcurrieron 3 años para que adoptaran innovaciones de cosecha y 16 años más para adoptar prácticas de establecimiento y manejo de la planta. En contraste, se observa que la innovación de administración, la adoptaron en el año 2000. Estos datos sugieren, que la adopción de innovaciones es gradual y en cadenas agroalimentarias como el café y otros productos, los productores lo hacen por imitación y principalmente aprenden entre familiares (Espejel, et al., 2019).

\subsection{Análisis de las redes de innovación}

El análisis de la red de innovación, identificó los actores que participan en el sistema de producción de café. Se registró una densidad baja de $2,1 \%$, lo cual indica que existe un escaso intercambio de conocimientos entre productores, situación que se presenta en otras cadenas productivas agroalimentarias como lo señala Espejel-García, et al. (2017). El indicador de centralización de entrada 
es de $92,17 \%$, lo cual indica que la red esta centralizada, es decir, predomina un actor o pequeño grupo de actores (productores), como se observa en la Figura II. Por otra parte, el grado de salida es de $3,55 \%$, significa que la búsqueda de información por parte de los productores es baja. Cabe destacar, que los productores referencian como fuente de innovación su propia experiencia (ExPro) y el conocimiento entre pares (Familiar).

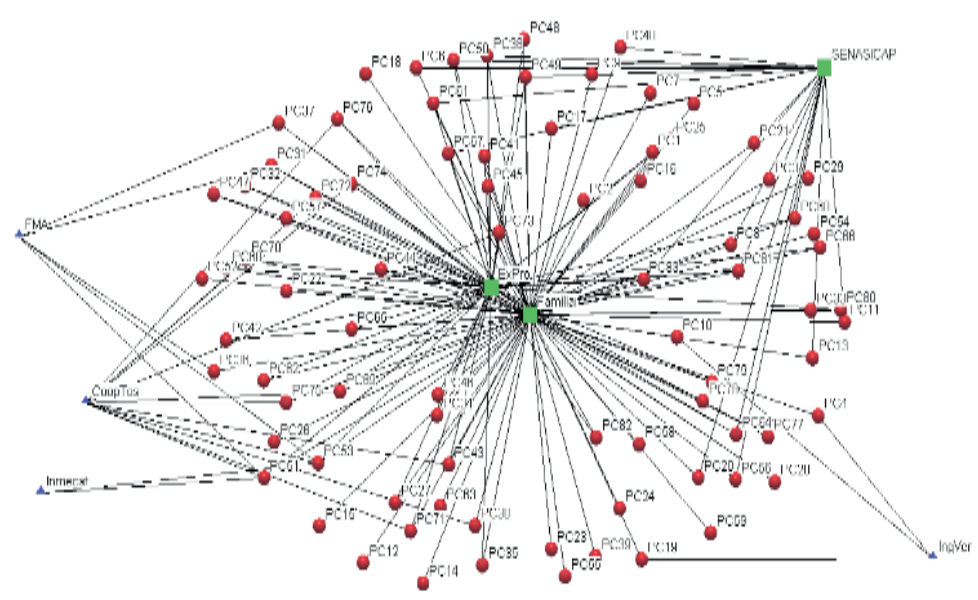

Fuente: Elaboración propia, 2020 con datos de la encuesta 2018.

Figura II: Red de innovación de café en la Sierra Norte y Nororiental de Puebla

Los resultados del análisis de componentes principales, muestran que un bajo porcentaje de productores ha adoptado innovaciones (Cuadrante III y IV) (ver Figura III). En el cuadrante II, los vectores indican que existe un nivel de asociación entre las innovaciones de control de maleza, reproducción y mejoramiento genético, establecimiento y manejo de plantación y nutrición, el grupo de productores ubicados en este cuadrante, priorizan la adopción de dichas innovaciones. En el cuadrante I, un reducido porcentaje de productores realiza las innovaciones de cosecha, sanidad y organización, cabe destacar que existe una mayor correlación de estas variables. 


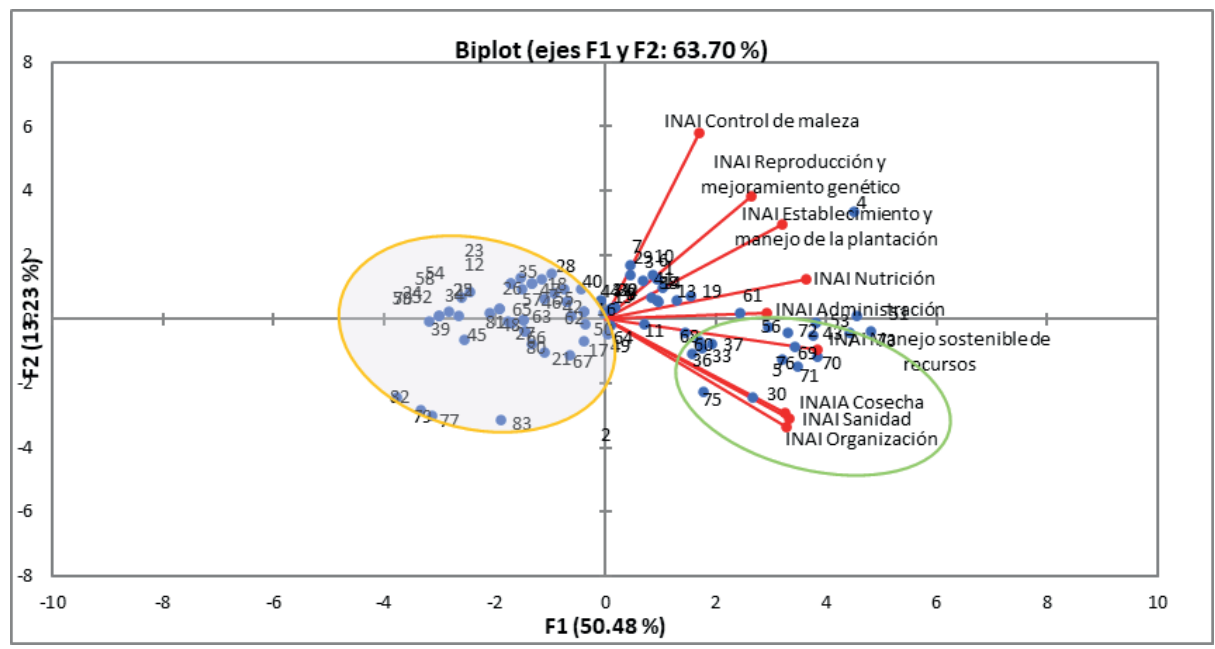

Fuente: Elaboración propia, 2020 con datos de la encuesta 2018.

\section{Figura III: Componentes Principales de los índices de adopción de innovación}

\subsection{Modelos de innovación en la comercialización de café}

La cooperativa Tosepan Titataniske (Unidos Venceremos) se fundó en 1974 a raíz de la problemática que existía con los altos precios del azúcar y maíz, así como el elevado intermediarismo en la comercialización del café, y que era manejado por aquellas personas de alto nivel socioeconómico; por ello, los pequeños productores obtenían beneficios marginales por la producción $\mathrm{y}$ venta de café. Tosepan Titataniske, es una unión de organizaciones integrada por ocho cooperativas que trabajan bajo objetivos en común, sin embargo, cada cooperativa opera de manera independiente y se somete a procesos de rendición de cuentas ante la asamblea general.

Maseual Xicaualis, es una de las ocho cooperativas que se encarga del acopio, beneficio, comercialización y asistencia técnica; inicio operaciones con la venta de productos convencionales: Café y pimienta, y en el año 2000 comenzaron con la producción y comercialización de los productos orgánicos, con el fin de incursionar en otros mercados con mejores precios y fue en el año 2003 cuando logró certificarse en la producción de café orgánico. Actualmente, la cooperativa cuenta con 684 socios que producen café, asociación café-pimienta, de diferentes comunidades de los municipios de Cuetzalan del Progreso, Zapotitlán, Huehuetla, Ixtepec, Jonotla, Hueytlalpan, Zoquiapan, Hueytamalco y Tepango de Rodríguez.

La cooperativa cuenta con certificaciones avalados por CERTIMEX, la cual certifica que el manejo de los cafetales y el beneficiado sean orgánicos, esta debe ser renovada cada año. También cuentan con el sello de comercio justo. Ambas distinciones, permiten a los productores exportar sus productos; asimismo, los inspectores realizan una estimación de cosecha en cada parcela para que de esta manera los productores entreguen la cantidad estimada a la cooperativa (Waridel, et al., 2001). Actualmente, la cooperativa exporta 


\section{Modelos de innovación en la producción de café en la Sierra Norte de Puebla-México}

Barrera Rodríguez, Ariadna; Ramírez García, Adán Guillermo;

Cuevas Reyes, Venancio y Espejel García, Anastacio

el café en pergamino a Japón, por medio de contratos que los productores realizan; en el año 2016, logró exportar 743 Qq de café pergamino y en el año 2016/17 exportaron $1.300 \mathrm{Qq}$ de café pergamino.

En el caso de que no cuente con la cantidad convenida, la misma cooperativa tiene relación con otras organizaciones del estado de Oaxaca y Chiapas, que producen café orgánico para acopiar y completar los lotes. Para que un productor sea parte de la cooperativa, tiene que solicitarlo ante la asamblea local, además, su parcela tiene que pasar por la etapa de transición que dura tres años, durante este tiempo el productor no debe aplicar ningún producto químico (fertilizante, herbicida, funguicida) a la plantación y debe realizar muestreo de suelo y de la planta, en caso de que las pruebas salgan positivas sobre la aplicación de un producto químico no podrá ser productor orgánico.

El productor orgánico debe ser: Comprometido y responsable para no afectar a los asociados de la organización. La misma cooperativa capacita a productores para que sean promotores y realicen inspecciones a la parcela y determinar si los productores llevan un manejo orgánico. También capacitan a los productores, para implementar diferentes innovaciones dentro de su parcela, como el manejo y conservación de los suelos, prevención y protección fitosanitaria en el que incluye la elaboración de biofertilizantes, caldo sulfocalcico, elaboración de compostas, entre otras.

Los productores, realizan el beneficio húmedo del café cuidando cada una de las etapas para no deteriorar la calidad del aromático (Ortega, 2010). La cooperativa, se encarga de acopiar el café pergamino orgánico, para ello cuenta con ocho centros de acopio en diferentes comunidades donde se ubican sus socios, con el fin de apoyar a los productores a reducir costos de transporte y a hacer eficiente los tiempos. Los acopiadores, son productores orgánicos que han sido propuestos por los mismos productores orgánicos de la comunidad, mismos que han sido capacitados por parte de la cooperativa para poder determinar la calidad del café de cada productor.

Los acopiadores, cuentan con una boleta de registro para la determinación de la calidad del café pergamino, con ella se dictamina si el café pergamino tiene buena, regular o mala calidad. Para identificar cada lote, se etiqueta cada saco de café pergamino, en ella se coloca los datos del productor, la calidad que obtuvo, número de sacos que entrega el productor, lugar de procedencia y tipo de zona: Alta, media o baja y posteriormente, se traslada a la cooperativa para el beneficiado seco.

La cooperativa Maseual Xicaualis, es la encargada de realizar el beneficiado seco que comienza a partir del mes de febrero y termina en junio. Realizan una segunda determinación de la calidad de acuerdo con la NMX-F-107SCFI-2008 Café verde en saco - muestreo. De toda la producción obtenida de café orgánica, el $80 \%$ se destina a mercados como Japón y Alemania, cumpliendo con los estándares de calidad para exportación, el $20 \%$ es destinado para la torrefacción y comercializado con la marca de la cooperativa -Tosepan Titataniskepara el mercado nacional y la marca -Maseualpara el mercado local, con el fin de que los productores consuman un café de calidad.

\subsection{Modelo de comercialización de café cereza y pergamino}

Para analizar la interacción que existe entre los diferentes actores (productorintermediario café) para la comercialización del café, ya sea en cereza o pergamino, se realizó un análisis de redes. La densidad de la red fue baja $(0,5 \%)$ un gran número de productores se encuentran aislados dentro de la red (nodos amarillos), es decir no venden su café. La red de comercialización de café cereza es centralizada y alrededor de esta existen subredes, los productores refieren a un solo actor (intermediario) para la venta de café cereza en cada municipio, mientras que el indicador de centralización de salida es un valor muy bajo, los productores no buscan a otros clientes para la venta de café (ver Figura IV). 

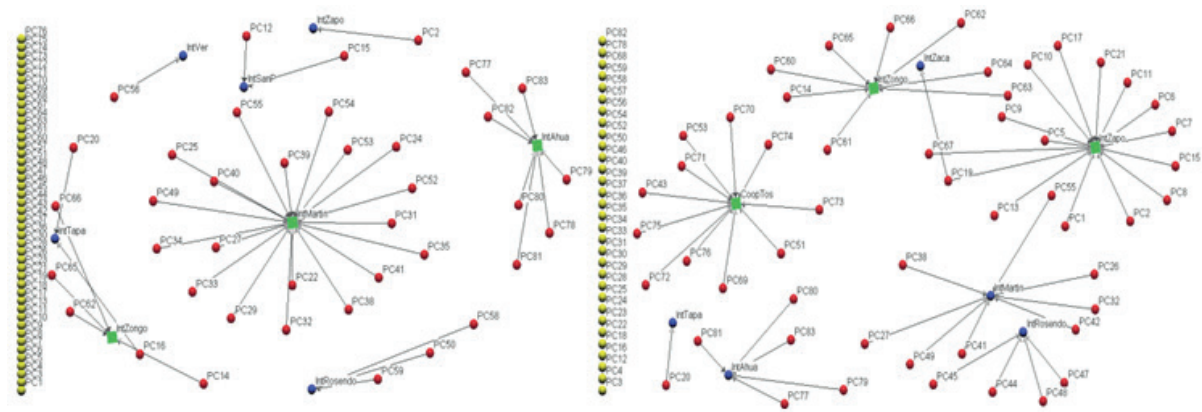

Café cereza

Café pergamimo

Fuente: Elaboración propia, 2020 con datos de la encuesta 2017.

\section{Figura IV: Modelo de comercialización de café cereza y pergamino de la Cooperativa}

La densidad de la red obtuvo un valor de $0,6 \%$, por lo que las relaciones entre los actores (productor-intermediario) es baja. La red de comercialización, se compone por pequeñas subredes, en la cual los productores refieren a los intermediarios y la Cooperativa Tosepan Titatiniske como compradores de café pergamino, ésta última cuenta con centros de acopio en las comunidades productoras de café. La CoopTos, IntZapo e IntZongo, son los principales actores que acaparan la producción de café pergamino con $38,64 \%$ en las comunidades de Zapotitlan y Zongozotla.

La cooperativa tiene convenios con diferentes instituciones entre ellas la Secretaria de Agricultura, Ganadería, Desarrollo Rural, Pesca y Alimentación (SAGARPA) hoy SADER; la Asociación Mexicana de la Cadena Productiva del Café A. C. (AMECAFÉ); la Universidad Intercultural del Estado de Puebla (UIEP) de Huehuetla; Universidad Autónoma Chapingo (UACh); y además forman parte del Sistema Producto de Café, Puebla. Los principales proyectos que tienen son capacitación a los productores para el manejo adecuado de los cafetales sostenible, obtención de semillas para el establecimiento de viveros, control de la roya (Hemileia Vastatrix) y broca (Hypothenemus Hampei Ferrari), secadores solares tipo invernadero para el secado de café y pimienta, entre otros.

\subsection{Percepción del consumidor en el modelo de comercialización}

Los resultados del análisis del consumidor, indican que el $73 \%$ son nativos de la Región Sierra Norte y Nororiental de Puebla y el $23 \%$ son de diferentes estados, entre ellos: Ciudad de México, Estado de México, Oaxaca, Veracruz, Hidalgo, Baja California y Chiapas. Los consumidores analizados tienen una edad que oscila entre los 14 a 91 años con un promedio de 46 años, para ellos, el café es la principal bebida después del agua, por lo que el consumo de café en los niños es habitual.

Los consumidores de café asociaron el termino café con cuatro palabras, entre ellos destacan: La familia, la convivencia y la amistad $(22,2 \%)$, puesto que al consumir una taza de café se puede tener una buena conversación entre familiares, amigos $\mathrm{o}$ compañeros de trabajo; economía y negocio 


\section{Modelos de innovación en la producción de café en la Sierra Norte de Puebla-México}

Barrera Rodríguez, Ariadna; Ramírez García, Adán Guillermo;

Cuevas Reyes, Venancio y Espejel García, Anastacio

$(19,9 \%)$ porque a los productores les genera ingresos que pueden satisfacer sus necesidades mediante un producto con gran demanda en el mercado y existen nichos de consumidores que están dispuestos a pagar por producto diferenciado; trabajo $(18,7 \%)$, por ser una planta que requiere de un buen manejo de la producción y también en la cosecha y postcosecha para garantizar una bebida de calidad; y alimento (15,8\%), es una bebida que se puede consumir en cualquier momento del día, principalmente durante la mañana.

Características organolépticas como es el sabor, aroma y color son atributos que definen la calidad del café. Con respecto a la cantidad de café que los consumidores compran el 71\% mencionó que adquieren menos de $1 \mathrm{Kg}$ a la semana y el $23 \%$ entre 1 y $2 \mathrm{Kg}$ de café molido. El 73\% mencionó que prefieren comprar el aromático en tiendas de abarrotes mientras que el $15 \%$ expresó que prefieren adquirir el aromático en tiendas especializadas como cafeterías. Un bajo porcentaje de consumidores conocen las marcas de café molido como Punta de Cielo, Bola de Oro, Sablan, Pasmar, el Nescafé clásico es la marca de café "soluble" que más conocen los consumidores por su disponibilidad en cadenas comerciales y tiendas locales, y las estrategias de mercadotecnia que desarrolla la empresa Nestlé.

Contrariamente, los consumidores entrevistados en la Expo Café 2018, consumen café de grano, los principales factores para que un consumidor cambie de marca son: La baja calidad del aromático, que no cuente con un sello distintivo, alto precio, por incumplimiento en la entrega y en menor proporción por salud.

De acuerdo con los datos obtenidos, el principal criterio que considera el consumidor para adquirir o consumir café, es el sabor (38\%), el aroma $32 \%$, el $16 \%$ expresaron que la calidad y $13 \%$ el precio del aromático. El consumidor busca un producto diferenciado de excelente calidad y que además cuente con diferentes certificaciones que garanticen su calidad y origen, por lo que está dispuesto a pagar un precio mayor con el propósito de deleitar su paladar con sabores únicos.
El café es una de las bebidas que contiene diferentes sabores, aromas, acidez, cuerpo, por lo que esto va a definir la calidad en taza (Regalado, 2006). Factores como la variedad de la planta, condiciones geográficas, el manejo del cultivo y del beneficiado, así como el almacenamiento y el grado de tueste, va a determinar su calidad. Los consumidores entrevistados mencionaron que el sabor y aroma son características esenciales que atraen al consumidor, mientras que la bebida al momento de consumirse debe estar caliente para degustar; otra característica que atrae al consumidor es el color, puesto que está relacionado con el grado de tueste.

La permanencia del sabor, amargo, dulce, y acidez, son características que percibe el consumidor. Con este trabajo de investigación, se encontró que los consumidores experimentan diferentes emociones, y la mayoría mencionó que se siente feliz, alegre, muy bien, también mencionaron que al consumir los mantiene despiertos, les proporciona fuerza o los mantiene activos durante el día. El café también les da tranquilidad y se mantienen relajados. Un estudio realizado señala que el café despierta una carga emocional positiva entre las personas que lo toman y su consumo se vincula, por este orden, a las emociones de placer, actividad, energía y felicidad.

Este estudio permite identificar nuevos nichos de mercado y modelos de comercialización, posibilita a las empresas el desarrollo de nuevos productos que proporcionen las experiencias más aceptadas y demandadas por los consumidores, es una herramienta imprescindible para diseñar acciones de comunicación y marketing (Espejel, et al., 2018). De acuerdo con el análisis de componentes principales los factores que inciden en el consumo de café se explican por el componente $1 \quad(39,4 \%)$ integrado por las variables socioeconómicas del consumidor como la escolaridad, ingreso, precio y etiqueta. El componente $2(22,7 \%)$ está compuesto por aroma y sabor, dos atributos sensoriales que determinan la calidad del café (ver Figura V). 


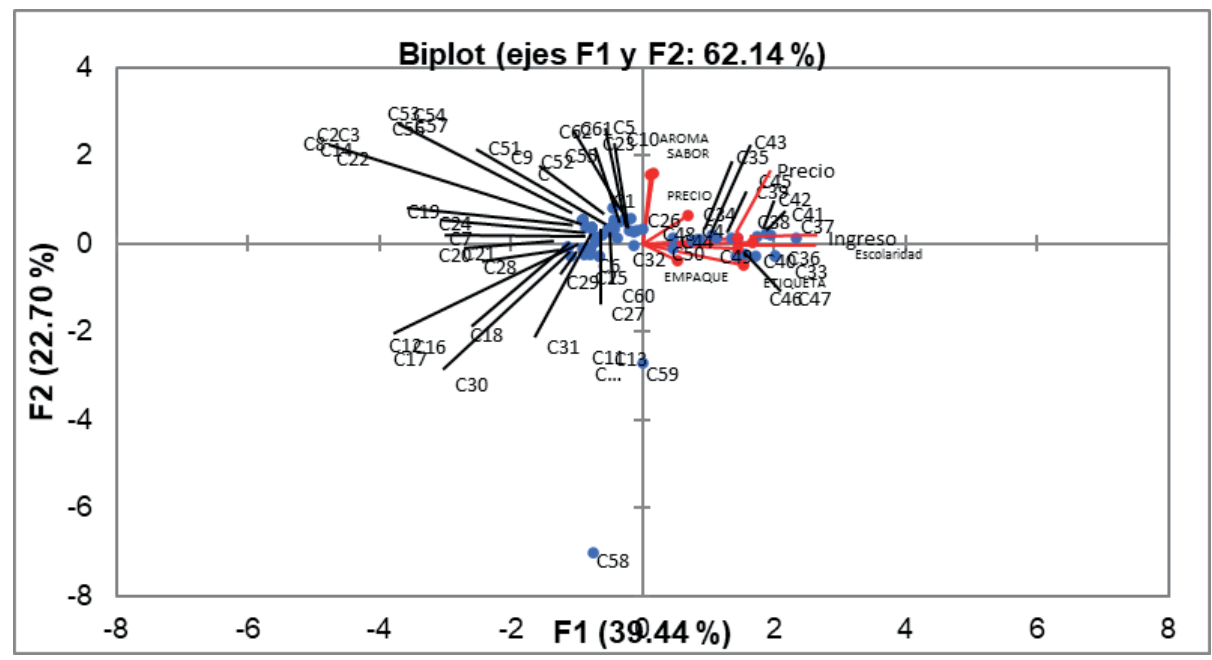

Fuente: Elaboración propia, 2020 con datos de campo, 2017.

Figura V: Diagrama de afinidad entre las variables y consumidores

\section{Conclusiones}

En la producción de café se han implementado modelos de innovación en el ámbito técnico-productivo, incentivados por la incidencia de enfermedades y plagas y la necesidad de incrementar la producción. Las innovaciones se transmiten mediante la capacitación y asistencia técnica; particularmente, en los modelos (estratos) de productores que registran mayor nivel de organización. Cabe destacar que se mantienen procesos de generación y transmisión de conocimientos entre pares (productores y familiares), existe un constante intercambio y transmisión de conocimientos tradicionales.

Los modelos de comercialización, son tradicionales por lo que es necesario innovar en función de las necesidades de los consumidores a fin de desarrollar nuevos nichos de mercado de mayor valor. Los modelos identificados están estrechamente relacionados con el trabajo de cohesión y organización que la cooperativa Tosepan ha desarrollado con los productores de café; por lo que este trabajo, evidencia la importancia de la organización para la producción y comercialización del café en regiones marginadas. A partir de los hallazgos de este estudio, se pueden implementar y desarrollar mecanismos de transferencia de tecnología con base en la funcionalidad e importancia de la organización en la Tosepan.

\section{Referencias bibliográficas}

Aguilar-Gallegos, N., Martínez-González, E. G., Aguilar-Ávila, J., Santoyo-Cortés, H., Muñoz-Rodríguez, M., y GarcíaSáncheza, E. (2016). Análisis de redes sociales para catalizar la innovación agrícola: de los vínculos directos a la integración y radialidad. Estudios Gerenciales, 32(140), 197-207. https:// doi.org/10.1016/j.estger.2016.06.006

Alburquerque, F. (2008). Innovación, 
Modelos de innovación en la producción de café en la Sierra Norte de Puebla-México Barrera Rodríguez, Ariadna; Ramírez García, Adán Guillermo;

Cuevas Reyes, Venancio y Espejel García, Anastacio

transferencia de conocimientos y desarrollo económico territorial: Una política pendiente. Arbor, 184(732), 687-700. https://doi.org/10.3989/ arbor.2008.1732.215

Aranda, J. G., González, B., y Reyes, S. T. (2017). Guía de buenas prácticas para café sustentable. Alianza Mexicoredd+. http://infocafes.com/ portal/biblioteca/guia-de-buenaspracticas-para-cafe-sustentable/

Blasco, T., y Otero, L. (2008). Técnicas conversacionales para la recogida de datos en investigación cualitativa: La entrevista (I). Nure Investigación, (33) Marzo-Abril

Canet, G., Soto, C., Ocampo, P., Rivera, J., Navarro, A., Guatemala, G. M., y Villanueva, S. (2016). La situación y tendencias de la producción de café en América Latina y el Caribe. Instituto Interamericano de Cooperación para la Agricultura (IICA) y Centro de Investigación y Asistencia en Tecnología y Diseño del Estado de Jalisco A. C. (CIATEJ).

Cuya, E. (2013). Guía técnica: Asistencia técnica dirigida en "cosecha y postcosecha en el cultivo de café". Agrobanco.

Escamilla, E. (1993). El café cereza en México tecnología de la producción. Universidad Autónoma Chapingo.

Escamilla, E. (2016). Las variedades de café en México ante el desafío de la roya. Breves de Políticas Públicas, (4).

Escamilla, E., Ruiz, O., Díaz, G., Landeros, C., Platas, D. E., Zamarripa, A., y González, V. A. (2005). El agroecosistema café orgánico en México. Manejo Integrado de Plagas y Agroecología (Costa Rica), (76), 5-16.

Escamilla, E., Ruiz, O., Zamarripa, A., y González, V. A. (2015). Calidad en variedades de café orgánico en tres regiones de México. Revista de Geografia Agricola, (55), 45-55.

Espejel, A., Barrera, A., Ramírez, G., y Cuevas, V. (2019). Innovación en la cadena agroindustrial Mezcal en Oaxaca, México. Revista Venezolana de Gerencia, 24(2), 188-209.

Espejel, A., Cuevas, V., Muñoz, M., Barrera, A., Cervantes, F., y Sosa, E. (2014). Sistema Regional de Innovación y Desarrollo Rural Territorial; pequeños productores de leche del Valle del Mezquital, Estado de Hidalgo, México. Spanish Journal of Rural Development, $V(2), 1-14$.

Espejel, A., Rodríguez, D. M., Barrera, A., y Ramírez, G. (2018). Factores estratégicos de la innovación y mercado en queserías artesanales de México. Revista Venezolana de Gerencia, 23(82), 424-441.

Espejel-García, A., Barrera-Rodríguez, A., Cuevas-Reyes, V., Ybarra, M. C., y Venegas, J. A. (2017). Sistemas de innovación y patrones de interacción local en el sector rural en México. Nova Scientia, 9(19), 595-614. https:// doi.org/10.21640/ns.v9i19.827

Fideicomisos Instituidos en Relación con la Agricultura - FIRA (2016). Panorama agroalimentario: Café 2016. FIRA. https://www.gob.mx/cms/uploads/ attachment/file/200636/Panorama Agroalimentario_Caf 2016.pdf

Fundación Produce Chiapas y Tecnológico de Monterrey (2003). Programa estratégico de necesidades de investigación y transferencia de tecnología del estado de Chiapas. Fundación Produce Chiapas y Tecnológico de Monterrey.

Galindo, G. (1995). Uso de innovaciones agrícolas en la región central de Zacatecas, México. Fitotecnia 
Mexicana, 18, 140-150.

Galindo, G. (2007). El servicio de asistencia técnica a los productores de chile seco en Zacatecas. Convergencia, 14(43), 137-165.

Inca Rural, SAGARPA, COFUPRO y CRUOUACh (2008). Serie cafeticultura: Manual del técnico cafetalero. Gestión de la calidad del café (catación) (8). INCA Rural, SAGARPA, COFUPRO, CRUO-UACh.

Instituto Mexicano del Café - INMECAFE (1974). Tecnología cafetalera mexicana: 25 años de investigación y experimentación. INMECAFE.

Instituto Nacional de los Pueblos Indígenas - INPI (2018). Acciones de gobierno para el desarrollo integral de los pueblos indigenas. Informe 2018. INPI. https://www.gob.mx/cms/ uploads/attachment/file/461777/inpiinforme-2018.pdf

Leal, M. E. (2012). Factores determinantes de la innovación tecnológica de las PYMES del sector confección. Revista de Ciencias Sociales (Ve), XVIII(3), 540-552.

Méndez, R. (octubre de 2006). Difusión de innovaciones en sistemas productivos locales y desarrollo territorial. III Congreso Internacional de la red SIAL, Alimentación y Territorios. Universidad Internacional de Andalucía, Jaen, España.

Muñoz, M., Aguilar, J., Rendón, R., y Espejel, A. (2007). Innovación: Motor de la competitividad agroalimentaria. Politicas y estrategias para que en México ocurra. CIESTAAM-UACh Fundación Produce Michoacán.

Muñoz, M., y Santoyo, V. H. (1996). Visión y misión agroempresarial: Competencia $y$ cooperación en el medio rural. Universidad Autónoma Chapingo.
Muñoz, M. y Santoyo, V. H. (2010). Del extensionismo a las redes de innovación. En J. Aguilar, J. Reyes y R. Rendón (Coords.), Del extensionismo agrícola a las redes de innovación rural (pp. 31-67). Universidad Autónoma Chapingo.

Navarro-Caballero, M., Hernández-Fernández, L., Navarro-Manotas, E., y HernándezChacín, J. (2020). Innovación en las micro, pequeñas y medianas empresas familiares del sector manufacturero del Atlántico-Colombia. Revista de Ciencias Sociales (Ve), XXVI(4), 124144. https://doi.org/10.31876/rcs. v26i4.34653

Organización de Cooperación y Desarrollo Económicos - OCDE y EUROSTAT (2005). Manual de Oslo: Guía para la recogida e interpretación de datos sobre innovación. OCDE y EUROSTAT. http://www.itq.edu.mx/ convocatorias/manualdeoslo.pdf

Ortega, M. T. (2010). Creación de un beneficio de café, en la congregación de El Troncal, Ver., para comercializarlo en café pergamino. Universidad Veracruzana Intercultural.

Regalado, O. A. (2006). ¿Qué es la calidad en el café? Universidad Autónoma Chapingo.

Rendón, R., Aguilar, J., Muñoz, M., y Reyes, J. (2007). Identificación de actores de clave para la gestión de la innovación: El uso de redes sociales. Universidad Autónoma Chapingo.

Santoyo, V. H., Díaz, S., Rodríguez, B., Pérez, J. R., Licona, A., Sosa, R., Valdivia, E., Sosa, L., y Márquez, C. (1994). Sistema Agroindustrial Café en México: Diagnóstico, problemática y alternativas. Centro de Investigaciones Económicas, Sociales y Tecnológicas de la Agricultura y la Agroindustria Mundial (CIESTAAM), Universidad Autónoma Chapingo. 
Santoyo, V. H., Díaz, S., Escamilla, E., y Robledo, J. D. (2016). Factores agronómicos y calidad del café. CENACAFE.

Servicio de Información Agroalimentaria y Pesquera - SIAP (2018). Datos sobre ciclo agrícola de café. SIAP.

Servicio Nacional de Sanidad, Inocuidad y Calidad Agroalimentaria - SENASICA y Secretaria de Agricultura, Ganadería, Desarrollo Rural, Pesca y Alimentación - SAGARPA (2017). Programa de trabajo anual de vigilancia epidemiológica del cultivo del café. SENASICA y SAGARPA. https://www.gob.mx/cms/uploads/ attachment/file/215280/PT VECC SLP 2017-.pdf
Vega, J. E., Bautista, M. D. C., y Martínez, M. D. C. (2020). Orientación emprendedora en la innovación de las pequeñas y medianas empresas en México. Revista de Ciencias Sociales (Ve), XXVI(4), 97-114. https://doi. org/10.31876/rcs.v26i4.34651

Velázquez, J. A., Cruz, E., y Vargas, E. E. (2018). Cooperación empresarial para el fomento de la innovación en la pyme turística. Revista de Ciencias Sociales (Ve), XXIV(3), 9-20.

Waridel, L., Meloche, F., Kirk, J., St-Pierre, R., Dueñas, R. M., Martínez, V. L., Campero, C., y VanderHoff, B. F. (2001). Un café por la causa hacia un comercio justo. Desarrollo gráfico. 